\title{
Trichorionic Triamniotic Triplets After Single Embryo Transfer: A Case Series and Literature Review
}

\author{
Isao Takehara ${ }^{1}$, Toshifumi Takahashi ${ }^{2 * \mathbb{D}}$, Kuniaki Ota $^{2}$, Nobuhiko Ohta ${ }^{3}$, Hideki Mizunuma $^{2}$, \\ Satoru Nagase ${ }^{1}$
}

\begin{abstract}
Objectives: Monozygotic triplet pregnancy very rarely occurs in assisted reproductive technology (ART) treatment. The present study reported two cases of trichorionic triplet pregnancies after single embryo transfer (SET) and reviewed the literature in this regard.

Case Presentation: In the first case, a 29 -year-old female underwent the long protocol using gonadotropin-releasing hormone analogue and one full blastocyst was transferred in a fresh cycle. At 6 gestational weeks, three gestational sacs were clearly observed, while only one fetus with fetal heart beat was found after one week. In the second case, a 39-year-old female underwent intracytoplasmic sperm injection treatment and seven embryos were frozen accordingly. After assisted hatching, one completely expanded blastocyst was transferred during a hormone replacement cycle. Three gestational sacs with three yolk sacs were observed at 6 gestational weeks. Finally, two fetuses with fetal heart beat were found after one week.

Conclusions: Overall, although the developmental mechanisms of monozygotic triplets are unknown, clinicians should be aware of the possibility of multiple pregnancies under SET.

Keywords: Triplets, Assisted reproductive technology, Monozygotic triplet, Single embryo transfer
\end{abstract}

\section{Introduction}

Assisted reproductive technology (ART) is widely used during infertility treatment. Overall, ART treatment increases the number of multiple pregnancies such as twins, triplets, and higher-order births (1). In addition, ART accounts for almost $80 \%$ of triplets and higher-order births (1). However, these multiple pregnancies should be prevented since they are associated with an increased risk of maternal and neonatal morbidity compared with twin and singleton pregnancies (2). Single embryo transfer (SET) is considered as the most effective strategy for preventing higher-order multiple pregnancies. Elective SET (eSET) is defined as the transfer of one good quality embryo in cases in which at least two good quality embryos are available. Nowadays, eSET markedly decreases the incidence of higher-order multiple pregnancies under ART treatment cycles (3). However, multiple pregnancies, including twin and triplet pregnancies are reported in some cases even when SET is performed to prevent their incidence (4).

In triplet pregnancies, the triplets can be of monozygotic, dizygotic, or trizygotic types. In the natural conception, the rate of monozygotic triplets varies from $13 \%$ to $26 \%$ whereas that of monozygotic triplets remains unknown after ART treatment (5). According to several reports, ART treatment increases the rate of monozygotic twinning compared with the natural conception $(4,6)$. Generally, it is difficult to determine the zygosity of triplet pregnancies by evaluating chorionicity and amnionicity instead of DNA by highly polymorphic marker analysis (5). In contrast, pregnancies after SET are monozygotic with some exceptions. Therefore, it was aimed to report 2 cases of trichorionic triplet pregnancies after SET, which were assumed to be monozygotic and to review the literature.

\section{Case Reports}

Case 1

A 29-year-old gravida 1 para 1 female referred to Yamagata University Hospital because of secondary infertility. She had undergone in vitro fertilization (IVF) and embryo transfer treatment because of tubal and ovulatory factors in the previous pregnancy. In addition, she suffered from oligomenorrhea, and a polycystic ovary was detected by transvaginal ultrasonography. Her husband's semen analysis showed normozoospermia based on the 2010 World Health Organization criteria. Therefore, she underwent three cycles of clomiphene citrate-stimulated intrauterine insemination without any success before undergoing IVF. Then, a long protocol with pituitary downregulation was followed with intranasal buserelin acetate and ovarian stimulation with the recombinant follicle-stimulating hormone. Human chorionic gonadotropin (hCG, $5000 \mathrm{IU})$ was administered 36 hours before oocyte retrieval (7). Then, 9 cumulusoocyte complexes (COCs) were retrieved by transvaginal 
ultrasound-guided aspiration, which were subjected to IVF, and seven out of nine oocytes were fertilized. Then, one complete blastocyst stage embryo with $3 \mathrm{BB}$ grade, according to Gardner's classification, was transferred 5 days after embryo culture. The patient had no unprotected sexual intercourse during the treatment period and her urine hCG was positive ten days after embryo transfer. In addition, three gestational sacs and one yolk sac were observed by transvaginal ultrasonography in the uterus at 6 gestational weeks whereas a fetus with heart beat was only found in one gestational sac containing a yolk sac at 8 gestational weeks (Figure 1a). However, no fetus was detected in the remaining two gestational sacs without yolk sacs. This was determined as vanishing triplets. The singleton pregnancy was uneventful, and she vaginally delivered a full-term (41 weeks) healthy female neonate (3010 g).

\section{Case 2}

A 39-year-old gravida 2 para 1 female attended Yume Clinic due to secondary infertility. Her previous two pregnancies were achieved by intracytoplasmic sperm injection (ICSI) because of male infertility caused by oligoasthenospermia. The short protocol of downregulation was followed with intranasal buserelin acetate and ovarian stimulation with recombinant follicle-stimulating hormone. hCG (10000 IU) was administered 36 hours before oocyte retrieval (7) and 11 COCs were retrieved by transvaginal ultrasound-guided aspiration. These COCs were denuded with $0.1 \%$ hyaluronidase and eight metaphase- 2 oocytes were subjected to ICSI. Of the eight oocytes, seven were fertilized and then cultured for five days. All developed embryos, including two cleavage embryos on day 3 and five blastocysts on day 5, were electively cryopreserved to avoid ovarian hyperstimulation syndrome. Three months later, one fully expanded blastocyst was warmed and assisted hatching was performed accordingly. One hatched blastocyst with 5AA grade according to Gardner's classification was transferred under hormone-replacement cycle. The patient had no unprotected intercourse during the treatment period. Ten days after embryo transfer, her urine hCG was found positive. Furthermore, three gestational sacs and three yolk sacs with two fetal heart beats were observed in the uterus at 6 gestational weeks by transvaginal ultrasonography. At 7 gestational weeks, three fetuses were detected in each gestational sac and the heart beat was observed in two fetuses of the three (Figure 1b). This was considered as vanishing triplets. The twin pregnancy was uneventful and she delivered healthy male neonates (2790 g \& $2596 \mathrm{~g}$ ) by elective caesarean section at 37 gestational weeks.

\section{Literature Review}

Related literature was reviewed using keywords such as "triplet", "triplets", "pregnancy", and "single embryo transfer" in PubMed and ICHUSHI database, which is a Japan Medical Abstract Service. During our search, only 12 cases were retrieved, including our cases on triplet pregnancy after SET (8-15). The characteristics of these cases are summarized in Table S1 (see Supplementary file 1). The leading cause of infertility was the male factor (in four cases, 33\%). Moreover, the number of cases with intracytoplasmic sperm injection (ICSI) and IVF was 6 (50\%) and 6 (50\%), respectively. Additionally, the median fertilization rates of IVF and ICSI were $85 \%$ and $56 \%$, respectively, indicating that the fertilization rate of IVF was significantly higher than that of ICSI by the MannWhitney $\mathrm{U}$ test $(P=0.02)$. There were $3(25 \%)$ and 9 (75\%) cases with cleavage embryo transfer and blastocyst transfer, respectively. Similarly, 4 (33\%) and 1 (8\%) cases were found with assisted hatching and embryo biopsy, respectively. The number of cases with oocyte and/or embryo with zona pellucida breaching (e.g., the one in ICSI, embryo biopsy, and assisted hatching) was 7 (58\%). Likewise, the number of cases with fresh and frozen embryo transfer cycles were $8(67 \%)$ and 4 (33\%), respectively. Nine cases were monochorionic triplets, which showed one gestational sac with three or four yolk sacs while, in one case, the female had 2 gestational sacs and three yolk sacs. However, three independent gestational sacs were observed in the cases of the present report. Five (42\%) cases in the literature review demonstrated a selective reduction of the fetus. Apart from our 2 cases, one case was observed with spontaneously vanishing triplets. Finally, the total number of cases with ongoing pregnancy and/or delivery was 10 (83\%).

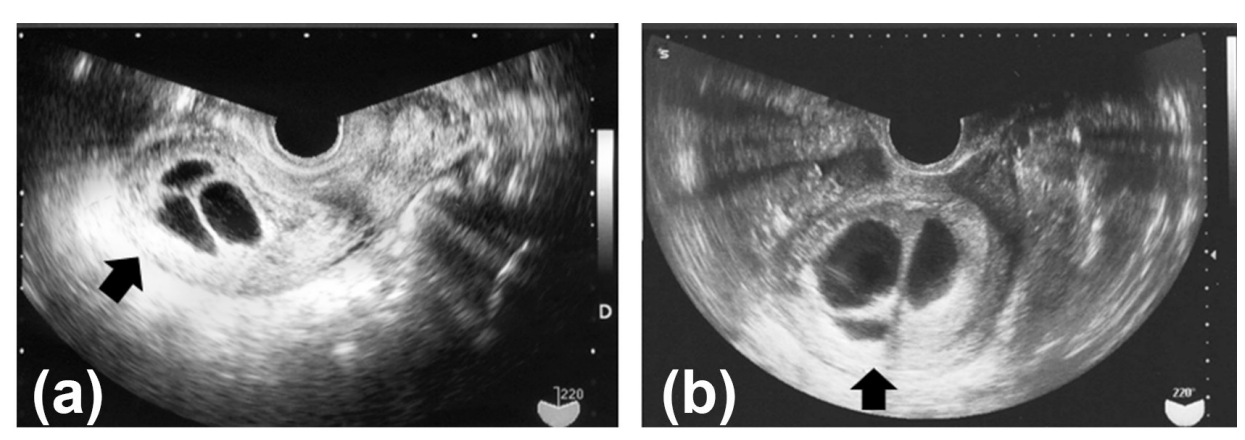

Figure. 1. Transvaginal ultrasonography of 2 Cases. Note. The arrows indicate three gestational sacs in the uterus: (a) Case 1 and (b) Case 2. 


\section{Discussion}

The current study was a report of two cases of triplet pregnancies after SET. Three independent gestational sacs were observed in the uterus of each case. These 2 cases of triplet pregnancies were considered as trichorionic pregnancies from monozygotic embryos. To the best of our knowledge, this is the first case report on trichorionic monozygotic triplets after SET.

Because there were no unprotected sexual activities, simultaneous spontaneous and ART-related pregnancy might not be considered (4). Therefore, both pregnancies were considered monozygotic triplets. In the literature review, there was no reported sexual intercourse during embryo transfer cycles except for our cases.

In addition, both cases might be trichorionic triamniotic triplets since three independent gestational sacs were observed in the present cases. In general, in twin pregnancy, a monochorionic twin occurs when an embryo divides at 4-8 days after fertilization while a dichorionic twin occurs when an embryo divides until 3 days after fertilization (16). A similar condition could be supposed in a triplet pregnancy as well. However, the determination of chorionicity and amnionicity is assumed to rely on natural conception. In the ART era, dichorionic diamniotic twins derived from SET were reported, which may be monozygotic $(4,17)$. Among the reports, single blastocyst transfer, which was cultured for 5 days after fertilization, resulted in dichorionic diamniotic twins. So far, this has not fit into the theory of embryo splitting timing and forming of monozygotic dichorionic twins. In the present cases, a blastocyst was transferred 5 days after fertilization whereas no division or splitting of the embryo was observed at that time. According to the long-held theory, the embryo should be monochorionic triamniotic triplets if it splits after the transfer. In fact, in the literature review, 9 out of 10 cases were monochorionic triplets with one gestational sac in each case. The transferred embryos were probably divided 4-8 days after fertilization. Further, as shown in monozygotic dichorionic diamniotic twins, embryo splitting possibly occurred after the embryo transfer in the present cases. Thus, the precise mechanisms or timing of embryo splitting for monozygotic multiple pregnancies remain unknown and the developmental mechanisms underlying monozygotic triplets are poorly understood as well. Monozygotic multiple pregnancies result from zygotic splitting and several ART procedures are probably considered as the risk factors for such splitting. Most studies regarding the risk factors of zygotic splitting after ART treatments are related to monozygotic twins. Younger maternal age seems to increase the rate of monozygotic twin pregnancies after ART treatment (4). As a matter of fact, the median age was 32 years in the literature review including our cases of triplet pregnancies. An earlier study reported that artificial breakage methods of zona pellucida and/or the plasma membrane such as those in ICSI, embryo biopsy, and assisted hatching are associated with the occurrence of monozygotic twins (18). Recently, big-data analyses of monozygotic twins have revealed that although fertilization methods (e.g., ICSI) are not a risk factor for zygotic splitting (4), assisted hatching is a risk factor for zygotic twin pregnancies (4). Many reports indicated that an extended culture of embryos to blastocysts is a strong risk factor for monozygotic twin pregnancies $(4,19)$. In the present study and in the literature review including our present cases, although the rate of artificial breakage of zona pellucida and/or the plasma membrane was 58\%, the rate of blastocyst transfer was as high as $75 \%$ in triplets after SET. There are conflicting reports regarding whether zygotic splitting is likely to occur in frozen-warmed embryo transfer cycles $(4,20)$. Furthermore, the number of frozen-warmed embryo transfer cycles was 4 of the 12 cases of triplets after SET in the present cases. Similar to the study of monozygotic twin pregnancies after ART, the above-mentioned ART procedures may increase the incidence of monozygotic triplets. Therefore, further studies are needed to determine how monozygotic triplets are formed in ART treatments.

In conclusion, we encountered very rare cases of trichorionic triplets after SET. Although the developmental mechanisms of monozygotic triplets remain unknown, clinicians should pay attention to the possibility of multiple pregnancies after SET.

\section{Conflict of Interests}

Authors declare that they have no conflict of interests.

\section{Ethical Issues}

This research program was approved by the Ethics Committee of Yamagata University Faculty of Medicine and informed consent was obtained from all individual subjects included in the study.

\section{Financial Support \\ None.}

\section{Supplementary Materials}

Supplementary file 1 contains Table S1.

\section{References}

1. Kulkarni AD, Jamieson DJ, Jones HW Jr, et al. Fertility treatments and multiple births in the United States. N Engl J Med. 2013;369(23):2218-2225. doi:10.1056/ NEJMoa1301467

2. Luke B, Brown MB. Maternal morbidity and infant death in twin vs triplet and quadruplet pregnancies. Am J Obstet Gynecol. 2008;198(4):401.e401-410. doi:10.1016/j. ajog.2007.10.785

3. Saito H, Jwa SC, Kuwahara A, et al. Assisted reproductive technology in Japan: a summary report for 2015 by The Ethics Committee of The Japan Society of Obstetrics and Gynecology. Reprod Med Biol. 2018;17(1):20-28. doi: $10.1002 / \mathrm{rmb} 2.12074$ 
4. Ikemoto Y, Kuroda K, Ochiai A, et al. Prevalence and risk factors of zygotic splitting after 937848 single embryo transfer cycles. Hum Reprod. 2018;33(11):1984-1991. doi:10.1093/humrep/dey294

5. Guilherme R, Drunat S, Delezoide AL, Oury JF, Luton D. Zygosity and chorionicity in triplet pregnancies: new data. Hum Reprod. 2008;24(1):100-105. doi:10.1093/humrep/ den364

6. Parazzini F, Cipriani S, Bianchi S, Bulfoni C, Bortolus R, Somigliana E. Risk of monozygotic twins after assisted reproduction: a population-based approach. Twin Res Hum Genet. 2016;19(1):72-76. doi:10.1017/thg.2015.96

7. Abdalla HI, Ah-Moye M, Brinsden P, Howe DL, Okonofua F, Craft I. The effect of the dose of human chorionic gonadotropin and the type of gonadotropin stimulation on oocyte recovery rates in an in vitro fertilization program. Fertil Steril. 1987;48(6):958-963. doi:10.1016/S00150282(16)59591-0

8. Risquez F, Gil M, D’Ommar G, Poo M, Sosa A, Piras M. Monochorionic triplets after single embryo transfer. Reprod Biomed Online. 2004;9(4):370-371. doi:10.1016/ s1472-6483(10)61270-9

9. Yanaihara A, Yorimitsu T, Motoyama H, Watanabe H, Kawamura T. Monozygotic multiple gestation following in vitro fertilization: analysis of seven cases from Japan. J Exp Clin Assist Reprod. 2007;4:4. doi:10.1186/1743-1050-4-4

10. Faraj R, Evbuomwan I, Sturgiss S, Aird I. Monozygotic triplet pregnancy following egg donation and transfer of single frozen-thawed embryo. Fertil Steril. 2008;89(5):1260. e1269-1212. doi:10.1016/j.fertnstert.2007.05.008

11. Lee SF, Chapman M, Bowyer L. Monozygotic triplets after single blastocyst transfer: case report and literature review. Aust N Z J Obstet Gynaecol. 2008;48(6):583-586. doi:10.1111/j.1479-828X.2008.00926.x

12. Dessolle L, Allaoua D, Freour T, et al. Monozygotic triplet pregnancies after single blastocyst transfer: two cases and literature review. Reprod Biomed Online. 2010;21(3):283289. doi:10.1016/j.rbmo.2010.04.011

13. Iwamoto H, Yoshida A, Suzuki H, et al. Monochorionic triamniotic triplet pregnancies with assisted reproductive technology: two case reports. J Obstet Gynaecol Res. 2010;36(4):872-875. doi:10.1111/j.1447-0756.2010.01235.x

14. Saravelos SH, Zhang T, Chung JP, et al. Monochorionic quadramniotic and triamniotic pregnancies following single embryo transfers: two case reports and a review of the literature. J Assist Reprod Genet. 2016;33(1):27-32. doi:10.1007/s10815-015-0611-2

15. Takeuchi S, Sugaya K, Murata A, Hase M, Suzuki T, Takakura T. A case of triplet pregnancy after single blastocyst transfer. Journal of Fertilization and Implantation. 2018;35(1):128131. [Japanese].

16. Hall JG. Twinning. Lancet. 2003;362(9385):735-743. doi:10.1016/s0140-6736(03)14237-7

17. Kyono K. The precise timing of embryo splitting for monozygotic dichorionic diamniotic twins: when does embryo splitting for monozygotic dichorionic diamniotic twins occur? Evidence for splitting at the morula/blastocyst stage from studies of in vitro fertilization. Twin Res Hum Genet. 2013;16(4):827-832. doi:10.1017/thg.2013.32

18. Skiadas CC, Missmer SA, Benson CB, Gee RE, Racowsky C. Risk factors associated with pregnancies containing a monochorionic pair following assisted reproductive technologies. Hum Reprod. 2008;23(6):1366-1371. doi:10.1093/humrep/den045

19. Hviid KVR, Malchau SS, Pinborg A, Nielsen HS. Determinants of monozygotic twinning in ART: a systematic review and a meta-analysis. Hum Reprod Update. 2018;24(4):468-483. doi:10.1093/humupd/dmy006

20. Mateizel I, Santos-Ribeiro S, Done E, et al. Do ARTs affect the incidence of monozygotic twinning? Hum Reprod. 2016;31(11):2435-2441. doi:10.1093/humrep/dew216

(c) 2019 The Author (s); This is an open-access article distributed under the terms of the Creative Commons Attribution License (http://creativecommons.org/licenses/by/4.0), which permits unrestricted use, distribution, and reproduction in any medium, provided the original work is properly cited. 